\begin{tabular}{|l|l|}
\hline $\begin{array}{l}\text { ESCOLA DE COMUNICAÇÃO, } \\
\text { ARTES E DESIGN } \\
\text { FUCRS }\end{array}$ & $\begin{array}{l}\text { FAMECOS } \\
\text { mídia, cultura e tecnologia } \\
\text { Revista FAMECOS, Porto Alegre, v. 28, p. 1-14, jan.-dez. } 2021 \\
\text { e-ISSN: 1980-3729 | ISSN-L: 1415-0549 }\end{array}$ \\
\hline https://dx.doi.org/10.15448/1980-3729.2021.1.35310
\end{tabular}

CIBERCULTURA

\title{
Streaming de conteúdo, streaming de si? Elementos para análise do consumo personalizado em plataformas de streaming
}

\author{
Streaming of content, streaming of oneself? Elements for analysis of personalized \\ consumption in streaming platforms \\ ¿Streaming de contenido, streaming en si mismo? Elementos para el análisis de \\ consumo personalizado en plataformas de streaming
}

\section{Sandra Portella \\ Montardo ${ }^{1}$}

orcid.org/0000-0001-8336-9329

sandramontardo@feevale.br

\section{Vanessa Amália \\ Dalpizol Valiati ${ }^{1}$ \\ orcid.org/0000-0001-9183-2301 \\ vanessavaliati@feevale.br}

Recebido em: 13 ago. 2019 Aprovado em: 2 maio 2020. Publicado em: 01 jul. 2021.

\section{(c) (1)}

Artigo está licenciado sob forma de uma licença Creative Commons Atribuição 4.0 Internacional.
Resumo: Este artigo tem por objetivo identificar elementos para a análise de consumo em plataformas de streaming pela Teoria de Prática (TP). Percebe-se que os sistemas de recomendação inerentes a essas plataformas produzem uma concepção de individualidade acionada por dinâmicas sociotécnicas que envolvem padrões de endereçamento de conteúdos e de anúncios combinados com os dados gerados pelas atividades dos usuários. Acredita-se que o caráter permanente dessa reciprocidade entre procedimentos automatizados e a ação dos usuários revela o consumo por meio de alianças temporárias entre essas partes e, por isso, renova o interesse nas abordagens de consumo que consideram a produção de sentido e de identidades. Aspectos materiais, competências, significados e dinâmicas relacionais são elementos provenientes das TP que servem como base para analisar consumo nas referidas plataformas.

Palavras-chave: Consumo. Plataformas de streaming. Teoria de Prática.

Abstract: This paper aims to identify elements for consumption analysis in streaming platforms through the Practice Theory. It is noticed that the recommender systems inherent to these platforms produce a concept of individuality driven by sociotechnical dynamics which involve addressing patterns of contents and ads combined with data generated by the users' activities. It is believed that the permanent character of this reciprocity between authomatized procedures and the users' action reveals the consumption through temporary alliances between both sides, reason by which it renews the interest in the consumption approaches which consider the production of meaning and identities. Material aspects, competences, meanings and relational dynamics are elements provided by the Practice Theory which work as a base to analyse consumption in these platforms. Keywords: Consumption. Streaming Platforms. Practice Theory.

Resumen: Este artículo tiene como objetivo identificar elementos para el análisis de consumo en plataformas de streaming a través de la Teoria de Práctica (TP). Se observa que los sistemas de recomendación inherentes a estas plataformas producen una concepción de la individualidad impulsada por dinámicas sociotécnicas que involucran patrones de direccionamiento, contenido y anuncios combinados con datos generados por la actividad del usuario. Se cree que la naturaleza permanente de esta reciprocidad entre los procedimientos automatizados y la acción del usuario revela el consumo a través de alianzas temporales entre ésos $y$, por lo tanto, renueva el interés en los enfoques de consumo que consideran el significado y la producción de identidad. Los aspectos materiales, las competencias, los significados y las dinámicas relacionales son elementos derivados de la TP, los cuales sirven como base para analizar el consumo en dichas plataformas. Palabras clave: Consumo. Plataformas de streaming. Teoria de práctica. 


\section{Introdução}

O streaming, enquanto transmissão de conteúdo via internet, vem alterando os usos cotidianos das mídias e das práticas da indústria midiática (BURROUGHS, 2015; SMITH; TELANG, 2016). Percebe-se que o investimento de grandes players da economia digital em plataformas de streaming, assim como sua popularidade entre os consumidores, é crescente. Dados do Music Consumer Insight Report 2018, pesquisa conduzida pela Federação Internacional da Indústria Fonográfica (IFPI) em vinte dos maiores mercados de música do mundo, indicam que $86 \%$ dos consumidores entre 16-64 anos acessam música e videos por serviços de streaming, sendo que o Brasil ocupa a terceira posição no consumo de música por esse tipo de plataforma (77\%). A Amazon, que comprou a plataforma de streaming de games Twitch, em 2014, está desenvolvendo a sua própria para lançamento previsto em 2020. ${ }^{2}$ No decorrer do quarto trimestre de 2017, a Netflix havia aumentado em $25 \%$ sua base de usuários, chegando a 117, 5 milhões, atingindo como valor de mercado a marca histórica de US\$ 100 bilhões. ${ }^{3}$

De acordo com Datta, Knox e Bronnenberg (2017), plataformas de streaming caracterizam-se por permitir acesso a uma vasta biblioteca de conteúdo a um preço fixo por mês ou, em certos casos, e sob determinadas condições, gratuitamente. O conteúdo pode ser disponibilizado sob forma de música (Spotify, Deezer, Tidal etc.), filmes/séries (Netflix, Amazon Prime Video, Hulu etc.), jogos (Twitch, HitBox, Beam, Azubu etc.), entre outras. Em relação à natureza e ao processo de acesso aos conteúdos desses catálogos, Vondereau (2015) destaca que a agregação de conteúdo passa a estar vinculada à escala e à diminuição da busca e dos custos de transação associados à economia digital por meio de novos sistemas para classificar, agregar e filtrar. Devido a isso, os serviços de streaming valem-se de dados gerados pelas atividades dos usuários, para direcionarem anúncios e moldarem seus sistemas de recomendação de conteúdo (PREY, 2018; CHENEY-LIPPOLD, 2017; ZUILHOF, 2017; DATTA; KNOX; BRONNEMBERG, 2017).

Ainda que de forma não muito recorrente, o consumo de mídia tem figurado como tema de estudo no âmbito da Teoria de Prática (WARDE, 2005: MAGAUDDA, 2011, MONTARDO, 2016; VALIATI, 2018). De acordo com essa abordagem, o interesse está em como a ação social é realizada e a constituição desses processos de atuação na vida social (HALKIER; JENSEN, 2011). Barbosa (2009) destaca a especificidade do consumo como mecanismo produtor de sentidos e de identidades, individuais e de grupo. Especificamente em relação aos estudos de mídia nesse contexto, Couldry (2010) destaca que é importante pesquisar a mídia a partir não apenas dos textos ou das instituições, como acontece nas pesquisas limitadas à audiência, mas também da prática voltada para entender o que as pessoas estão fazendo em relação à mídia em uma gama de situações e contextos diferentes. A pergunta que se coloca, então, é: que elementos devem ser levados em conta para compreender "o que as pessoas estão fazendo" quando a mídia em questão são plataformas de streaming de modo que suas práticas são totalmente atravessadas por procedimentos automatizados, desconhecidos em sua totalidade, que não só moldam essas mesmas práticas, mas que, devido a isso, também reconfiguram permanentemente as próprias plataformas? Em se tratando desse tipo de plataforma, o que deve ser levado em conta para captar os efeitos da personalização desses serviços na vida de seus usuários?

Frente a isso, este artigo tem por objetivo propor um modelo que contemple elementos-chave para a análise de consumo em plataformas digitais de streaming. Para tanto, faz-se necessário, primeiramente, verificar as particularidades do consumo nesse tipo de plataforma. Em seguida, investiga-se a pertinência da Teoria de Prática na abordagem de consumo digital entendido como usos de

\footnotetext{
2 Disponivel em: https://tecnoblog.net/27479g/amazon-streaming-jogos. Acesso em: 11 fev. 2019

Disponivel em: https://canaltech.com.br/resultados-financeiros/netflix-base-de-usuarios-cresce-25-e-servico-passa- a-valer-us-100-bilhoes-107028. Acesso em: 11 fev. 2019.
} 
plataformas de streaming. Com isso, acreditamos que reunimos subsídios teóricos suficientes para identificar elementos que tornem possivel a análise de práticas ai realizadas e do seu entrelaçamento na vida social contemporânea. O estudo se vale de pesquisa bibliográfica e documental.

\section{Consumo personalizado em plataformas de streaming}

De acordo com Thomes (2011), streaming envolve: a) consumo de bens informacionais sem que, necessariamente, haja a posse do arquivo do conteúdo; b) armazenamento de arquivos em um servidor; c) acesso de arquivos sob demanda. Ainda segundo Thomes (2011), um dos tipos de fonte de receita desse tipo de plataforma são assinaturas mensais, que garantem de acesso "ilimitado" ao catálogo em questão (Netflix, Hulu, Amazon Prime, YouTube Prime, Spotify, Deezer e Tidal). Outro tipo é a publicidade que, em serviços de streaming de música e de games, subsidia o acesso gratuito do catálogo por meio de anúncios que interrompem a experiência de consumo. Em plataformas de streaming de games, como o Twitch, a assinatura mensal pode liberar o acesso de determinados recursos não disponiveis gratuitamente (MONTARDO; PAZ, 2017).

Obviamente, os usos de streaming não se restringem ao acesso a esse tipo de plataforma. O acesso de vídeos em geral e de videoclipes na versão gratuita do YouTube, ou mesmo pelo Facebook, por exemplo, contempla as caracteristicas nomeadas por Thomes (2011). Prey (2018) argumenta que música é um campo fértil para que os usuários percebam a si próprios enquanto indivíduos, uma vez que está conectada com nosso cotidiano, nossa memória, nossas aspirações e nossa identificação com determinados grupos sociais (FRITH, 1998; BOURDIEU, 1984). O mesmo poder-se-ia dizer de filmes/séries e jogos digitais. Portanto, o conteúdo deve ser primordial na delimitação das plataformas de streaming que interessam ao modelo a ser proposto, principalmente, pelo fato de que uma série de camadas de software e hardware acabam por delinear as "condições de possibilidade" (CHENEY-LIPPOLD, 2017) de alcance daquela impressão de individualidade por meio do acesso e da manipulação desses conteúdos.

Vonderau (2015) aborda o aspecto de agregação de conteúdo inerente às plataformas de streaming, descrevendo a agregação como a capacidade de extrair conteúdo de várias fontes e torná-lo acessivel em um servidor específico. O autor ressalta que locais de agregação cultural existiram muito antes da internet (bibliotecas, museus, arquivos etc.). No entanto, continua o autor, a agregação também entrou em um vocabulário conceitual mais específico, vinculado à escala e à diminuição da busca e dos custos de transação associados à economia digital. "A agregação representa uma tendência tecno-social que combina um alcance cada vez maior de cultura, conhecimento e commodities com novos sistemas para classificar, agregar e filtrar [...]" (VONDERAU, 2015, p. 720).

Nesse sentido, Burroughs (2015) ressalta que com o aumento da largura de banda da rede no início dos anos 2000, o streaming tornou-se essencial não apenas às práticas de negócios, mas também à forma como encontramos e habitamos a cultura mediada (como por exemplo, o consumo atual de entretenimento). Dessa forma o streaming é tanto tecnologia quanto uma prática cultural que configura o público e a indústria. Com relação às características técnicas do sistema de streaming, Burroughs (2015) explica que os dados digitais são extremamente maleáveis e podem ser armazenados, compartilhados e distribuidos. Para o autor, o streaming como uma prática tecnológica não foi possivel até que os dados pudessem ser comprimidos e descomprimidos para permitir que os usuários armazenassem e simultaneamente consumissem conteúdo. Isso permitiu que a informação digital fosse flexivel e manipulável à medida que os dados atravessam as múltiplas plataformas e formatos mediados. Com base no trabalho de Flew (2002), Burroughs (2015) também destaca o caráter networkable (facilmente distribuída em rede), compressivel (diminuição da quantidade de largura de banda necessária para enviar pacotes de dados descontínuos através da inter- 
net) e denso (muita quantidade de informações armazenadas em um determinado espaço) dos sistemas de streaming. Burroughs (2015) aponta ainda duas outras propriedades adicionais da midia digital que podem ser aplicadas ao streaming: a primeira, a automação, que permite que os algoritmos manipulem a composição numérica dos dados digitais sem intervenção humana - o autor utiliza como exemplo o sistema de recomendação da Netflix, que filtra automaticamente as entradas do usuário e gera recomendações que reforçam o fluxo no streaming. A segunda propriedade é a possibilidade da construção de bancos de dados que armazenam, filtram e recuperam dados dos usuários.

Entre os estudos inspirados nessas particularidades, destacam-se os referentes ao impacto das plataformas de streaming na indústria do entretenimento em termos de produção, consumo e distribuição de conteúdo (SMITH; TELANG, 2016; THOMES, 2011; ALILOUPOUR, 2016; SLAGER, 2016; COFFEY, 2016; SHARMA, 2016). As produções que focam os usos dessas plataformas são os que mais interessam ao escopo deste artigo. Elas abordam de maneira mais enfática as implicações dos modos de personalização no acesso aos conteúdos aí disponibilizados que são, via regra, problematizados pelos seus autores. Em relação aos objetos de estudo, com exceção de Burroughs (2015), que explora Netflix, todas as demais produções se aplicam a serviços de streaming de música, como será mostrado a seguir.

Hagen e Lüders (2016) investigam música como objeto social via compartilhamento de arquivos ou ação de seguir usuários no Spotify e no Tidal, e identificam questões de gerenciamento de performance nessas ações. A partir de 23 entrevistas em focus-group com 124 usuários das referidas plataformas, diários sobre a prática de ouvir música, observação on-line e entrevista com 12 heavy-users, as autoras concluem que a negociação empreendida em torno do consumo de música nos serviços de streaming analisados variam entre usos pessoal e social, com gradações no segundo tipo. Pode-se dizer que esse estudo presume a ação dos algoritmos por meio de múltiplos métodos de acesso à experiência dos usuários, privilegiando, em sua análise, as impressões emergentes quanto a isso a partir dessas experiências e não, de forma complementar, via exploração das plataformas em si. De forma similar, Datta, Knox e Bronnenberg (2017), não se aprofundam na mediação dos algoritmos nos serviços analisados em artigo centrado nas mudanças no comportamento de escuta de música, relativas ao aumento de variedade de canções e de gêneros musicais e facilidades de descoberta de novas músicas viabilizadas pelo Spotify.

Com o objetivo de analisar as práticas da audiência com Netflix, a partir de enquadramento conceitual de Certeau (1984), Burroughs (2015) questiona a ideia de "audiência algoritmica", caracterizando-a, antes, como um endereçamento discursivo estratégico para que essa plataforma de streaming se diferencie como produtora entre outras do mercado audiovisual, uma vez que se basearia em dados coletados para prever o tipo de produto esperado pela audiência, além de, pelo mesmo motivo, supostamente assegurar que os usuários estariam pagando apenas pelo que gostariam de ver. Segundo o autor (2015), a não disponibilização dos dados de audiência de seus produtos pela companhia são um indicativo de sua dúvida, o que o leva a concluir que algoritmos e dados podem operar, predominantemente, ao menos, em um nivel discursivo.

Dois estudos se centram no processo de "individuação algoritmica" em plataformas buscando caracterizar o que personalização significa nesses contextos. Originalmente, o termo é proposto por Zuilhof (2014) em The soudtracked self: algorithmic individuation on Spotify. Zuilhof (2014) analisa o processo de curadoria de música efetuada pelo sistema de recomendação no Spotify e de como esse processo influencia a individuação, a partir de aporte teórico marxista. Conceito central para Zuilhof (2014) é o de prosumption que designa não um ato individual, mas um agregado de atividades coletivas dentro do sistema, incluindo produção, distribuição e consumo.

Em Nothing personal: algorithmic individuation on music streaming platforms, Prey (2018) objetiva 
identificar modos de perceber o ouvinte operados pela Pandora Internet Radio e pelo Spotify. Em face dos sistemas de recomendação das referidas plataformas, que visam direcionar anúncios de forma personalizada para os seus usuários, ao mesmo tempo que definem o tipo de música a ser disponibilizada para cada um deles, Prey (2018) se vale da teoria da individuação de Gilbert Simondon (2009), que prevê que o individuo seja antes um efeito de individuação do que uma causa, de modo que está em constante processo de se tornar individuo, para examinar, justamente, o processo de personalização desses serviços. Isso porque com as músicas que ouvimos, os filmes/séries que assistimos, as compras que fazemos, "nós produzimos nossa identidade, nós modulamos a nós mesmos como indivíduos" (PREY, 2018, p. 10).

Com vistas a identificar os critérios empregados para construção dos sistemas de recomendação do Spotify e, consequentemente, dos perfis musicais dai advindos, Prey (2018) explora o recurso Discover Weekly, responsável por indicar 30 novas faixas por semana baseada no gosto musical de seus ouvintes, com o que identifica um sistema híbrido de recomendação. Segundo Prey (2018), esse sistema é abastecido por, um lado, por um software de análise acústica (The Echo Nest) que classifica as canções de acordo com múltiplos fatores aurais, de modo que cada canção seja interpretada a partir de, em média, dois mil "eventos" (dançabilidade, "tempo" etc.); e, por outro, através da análise semântica de conversações on-line sobre música que, por meio da relação entre palavras-chave e frases, produz efeitos de similaridades em um nível "mais cultural". Esses dados abastecem o Taste Profile, ferramenta de análise e visualização de preferências, que constituem um perfil musical baseado em gosto musical (seleção de faixas e de artistas) e comportamento musical (favoritas, rankings, atitude de pular faixas etc.).

Além disso, as playlists propostas pelo próprio Spotify, bem como as playlists elaboradas pelos ouvintes com mais seguidores, são privilegiadas no sistema de recomendação do Discover We- ekly, afirma Prey (2018) ao citar Pasick (2015). A partir desse conjunto de processos, o sistema filtra as músicas que ainda não figuram no perfil do ouvinte e as recomenda, a partir das concepções de similaridade que daí são deduzidas. Nas palavras do autor: "Para o Spotify, o indivíduo não é entendido em relação ao objeto musical, mas em relação ao seu comportamento de escuta anterior, e com outros individuos considerados mais similares" (PREY, 2018, p. 1091). Essa percepção é condizente com a abordagem de produção de perfis em ambientes digitais de Bruno (2013) que a concebe antes como expressão de relações interpessoais do que com as intrapessoais.

Sistemas de recomendação não levam em conta marcadores fixos de identidade (critérios demográficos, por exemplo), mas sim "modelos de comportamento flexiveis" (CHENEY-LIPPOLD, 2017). Uma expressão pragmática desse tipo de modelo é o fornecido pelo que Pagano et al. (2016) identificam como "virada contextual dos sistemas de recomendação" que se opõe ao Paradigma de Preferência Imutável (ImP). Esse paradigma, que não prevê a possivel mudança de gostos e objetivos dos usuários, opõe-se à virada contextual em sistemas de recomendação, que interpreta que somos mais parecidos com quem tem os mesmos objetivos que nós do que com versões antigas de nós mesmos (PAGANO et al., 2016), com o que se conclui que sistemas de recomendação não consistem em recomendação para um único indivíduo.

Da mesma forma, Bruno (2013) afirma que a noção de individualidade atrelada à individualização algoritmica consiste na concepção de perfis no ambiente digital de acordo com as possibilidades de extração de conhecimento a partir deles, referindo-se mais ao futuro do que ao passado, mais à exterioridade do que à interioridade, consistindo menos em singularidade do que em regras de similaridade. Cheney-Lippold (2017) expressa esse ponto por meio do conceito de "tipos mensuráveis", uma espécie de caricatura concebida pelos dados considerados úteis para as categorizações requeridas em diferentes plataformas digitais, resultantes das 
atividades on-line empreendidas pelos usuários. Assim, esse usuário é interpretado pelas redes de computadores, pelos governos e pelos seus amigos, temporariamente, como membro de diferentes categorias emergentes.

Nesse sentido, a partir de algoritmos sensiveis ao contexto, que rastreiam comportamento por sinais advindos de vários dispositivos móveis, torna-se viável coletar e agregar dados dos ouvintes em relação a hábitos durante o dia, conteúdos consumidos e estado emocional, o que se reflete na definição de públicos-alvo para anunciantes. A propaganda tradicional não se dirige a um indivíduo, mas a públicos-alvo. O que chama a atenção nas plataformas analisadas por Prey (2018) é o nivel de detalhe e o grau de fragmentação com que esses públicos são projetados, como por exemplo, ouvintes hispânicos, ouvintes hispano-falantes, pela Pandora Radio, ou de acordo com suas atividades diárias, conforme o Spotify. Os Branded Moments, modalidade de patrocínio de playlists do Spotify, disponiveis mesmo em assinaturas pagas, disponibiliza as oportunidades de as marcas patrocinarem listas de músicas concebidas para treinos, festas, ou hora de dormir, por exemplo, e, ainda, permite fragmentar cada momento sob forma de proposição de playlists supostamente adequadas a eles (por exemplo, diferentes tipos de festa).

Finalmente, Prey (2018) propõe que a individuação algoritmica, tal como observado nas plataformas de streaming por ele analisadas, designa um processo sociotécnico dinâmico acionado para atualizar o indivíduo por meio de seu uso, com o que evidencia um potencial de identidade processual afetado diretamente pelos interesses financeiros das plataformas e de seus anunciantes.

A partir desse conjunto de estudos, conclui-se que a investigação sobre o consumo em plataformas de streaming requer, necessariamente, a problematização da ideia de personalização inerente a esses serviços, via exploração da performance algoritmica implicada nas práticas dos consumidores dos conteúdos nelas disponibilizados para posterior exame dos efeitos desse conjunto de procedimentos automatizados nas percepções dos usuários sobre si e sobre os outros. A seção a seguir busca apresentar a Teoria de Prática de modo a identificar em que medida suas abordagens são pertinentes para viabilizar esse tipo de análise.

\section{Consumo e teoria de prática}

A teoria de prática (TP) é um ramo da teoria social centrado em práticas em vez de estruturas, sistemas, indivíduos ou interações (POSTILL, 2010). Essa teoria não representa um campo unificado e coerente de estudos, mas um conjunto de textos produzidos por pensadores com diferentes versões de leituras teóricas que têm em comum o foco em como a ação social é realizada e a constituição destes processos de atuação na vida social (HALKIER; JENSEN, 2011). Devido à multiplicidade do corpo teórico, é importante ressaltar que este artigo privilegia a abordagem na qual as práticas são a configuração de uma série de dinâmicas interconectadas (HALKIER; JENSEN, 2011). Tal como define Reckwitz (2002), compreende-se a prática como:

Um tipo de comportamento rotinizado que
consiste em vários elementos interconectados
entre si: formas de atividades corporais, formas
de atividades mentais, 'coisas' e seus usos, co-
nhecimento prévio na forma de entendimento,
habilidade para saber fazer algo, estados de
emoção e conhecimento motivacional. Uma
prática - jeito de cozinhar, de consumir, de
trabalhar, de investigar, de tomar conta de si
ou de outros - forma, por assim dizer, um bloco
cuja existência depende necessariamente da
existência e da interconexão especifica desses
elementos, e não pode ser reduzido a qual-
quer um destes elementos individualmente
(RECKWITZ, 2002, p. 249).

Schatzki (2001), um dos autores mais populares desta geração de teóricos, apresenta a ideia de um "campo de práticas", no qual estão inseridas a atividade humana, a ciência, a linguagem, as instituições sociais e as transformações históricas, e que pode ser definido como "o nexo total de práticas humanas interligadas" (2001, p. 12). Assim, a prática pode ser considerada como um conjunto de ações enquanto atividades organizadas, que podem ser interpretadas tanto como uma entidade coordenada quanto como performance 
(SCHATZKI, 2001, 1996). Nesse sentido, para que os fazeres e dizeres que compõem uma prática constituam um nexo é preciso que eles estejam conectados. Shove, Pantzar e Watson (2012), seguindo a perspectiva de Schatzki $(2001,1996)$, reconhecem a prática enquanto performance e entidades coordenadas, mas acrescentam que elas são definidas pelas relações interdependentes entre materiais (objetos, tecnologias, entidades fisicamente tangiveis, e coisas das quais os objetos são feitos), competências (habilidades, know-how e técnicas) e significados (que inclui significados simbólicos, ideias e aspirações).

No campo midiático, de acordo com Postill (2010) essa abordagem oferece aos estudos da mídia novas formas de tratamento para questões centrais ao campo, em eixos como a mídia no cotidiano, mídia e o corpo, e a produção de mídia. A mídia, para o autor torna-se um campo de observação do jogo cultural cotidiano de reprodução e mudança, em que praticantes (com diferentes graus de saber-fazer, disciplina e comprometimento), práticas e tecnologias migram e circulam através das fronteiras desse campo. Sob esse aspecto, o consumo, sob o viés da teoria da prática, pode ser entendido como um fenômeno que se manifesta como um conjunto de práticas (CASTAÑEDA, 2010). Os estudos que relacionam a teoria da prática ao consumo baseiam-se na aproximação realizada por Warde (2005), para quem o consumo é o

[...] processo pelo qual os agentes envolvem-se na apropriação e apreciação, seja por razões contemplativas, expressivas ou utilitárias, de bens, serviços, performances, informação ou ambiente, tenham eles pago por isso ou não, sobre os quais o agente tenha algum grau de poder. Desse modo consumo não é uma prática em si, mas um momento de quase toda prática (WARDE, 2005, p. 137)

Nessa perspectiva, "o consumo ocorre dentro e por causa das práticas" (WARDE, 2005, p. 145). A prática, explica Warde (2005), requer o consumo de determinados bens e serviços, e exige que os praticantes tenham a capacidade de manipular as ferramentas apropriadas, dedicando um nivel adequado de atenção à sua condução, além de exibir entendimento comum, know-how e engajamento. Para o autor, esse engajamento, muito mais que qualquer decisão pessoal, é o que explica a natureza e o processo de consumo. A prática, nesse caso, é o canal para as gratificações que surgem nos momentos que compõem o consumo. Essa gratificação está mais ligada ao desempenho de determinada prática, que requer a posse ou acesso a determinados serviços, do que ao ato de consumir propriamente dito.

Para a TP os objetos são componentes necessários e tão indispensáveis quanto as atividades mentais e corporais (RECKWITZ, 2002). Assim, evidencia-se que para uma análise mais ampla do cenário do consumo em plataformas de streaming torna-se necessário também compreender a materialidade como um dos elementos que interagem com os processos de surgimento e transformação das práticas através da integração de novos objetos e/ou tecnologias (MAGAUDDA, 2011). Essa integração se dá, via de regra, por meio do consumo (de bens ou de conteúdos). A observação das affordances, competências e significados da plataforma e do conteúdo no campo de práticas dos usuários torna possivel a visualização dos nexos arranjo-práticos (SCHATZKI, 2010) e características desse tipo de consumo, como por exemplo a presença dos algoritmos e diferentes suportes como elementos que atuam como mediadores do processo e o estabelecimento de fluxos específicos de consumo.

Sob esse aspecto, Lemos (2013, p. 19) ressalta que na cultura contemporânea, mediadores não-humanos, 4 "nos fazem fazer (nós, humanos) muitas coisas provocando mudanças em nosso comportamento no dia a dia e também, em contrapartida, recursivamente, mudamos esses não humanos de acordo com as nossas necessidades". No caso da comunicação, continua o autor, a mediação ${ }^{5}$ está inserida na materialidade dos dispositivos, na constituição do seu design -

\footnotetext{
4 Para o autor, exemplos de mediadores não humanos: objetos inteligentes, computadores, servidores, redes telemáticas, smartphones, sensores etc.

5 A mediação é um conceito da Teoria Ator-Rede. Lemos (2013, p. 48) resume a "toda ação que um actante faz a outro, implicando ai estratégias e interesses próprios na busca da estabilização futura da rede ou da resolução da estratégia ou do objetivo".
} 
assim, a mediação dos artefatos digitais torna-se hoje parte intrínseca do nosso cotidiano.

Em certo sentido, pode-se associar essa perspectiva a de neomaterialidade tal como proposto por Paul (2015) no contexto da arte e das tecnologias digitais. Segundo a autora, neomaterialidade descreve, em primeiro lugar, a confluência e a convergência das tecnologias digitais em várias materialidades, e, em seguida, as formas pelas quais essa fusão modificou nossa relação com essas materialidades, assim como a nossa representação como sujeitos. Com isso, torna-se fundamental considerar as afetações mútuas entre humanos e não-humanos, conforme posto anteriomente, para que se obtenha uma compreensão mais ampla do consumo de streaming no âmbito da TP.

É possivel relacionar o consumo em plataformas de streaming às três superficies materiais indispensáveis elencadas por Thrift (2005): a tela, o software e o corpo. As telas já são uma constante nas atividades do cotidiano: a existência de smartphones, computadores, televisão, cinema, dentre os mais variados artefatos estão ligados a este suporte, cuja função está centrada em comunicar, afetar, entreter, funcionando como uma vitrine do pensamento (THRIFT, 2005). O software, a segunda superficie, é abordada por Thrift (2005) como um conjunto de novas textualidades (programação, linguagem, serviços), que, com seus próprios protocolos e regras, guarda relação com as telas. O autor ressalta que, assim como as telas, ele também se faz cada vez mais presente no cotidiano dos individuos, ocupando microespaços e gerando uma sensação de familiaridade, ao mesmo tempo em que ficam em um "estado de fundo", tornando-se imperceptíveis. Com relação ao corpo, ele é considerado pelo autor mapa das mudanças sociotécnicas que se vive na contemporaneidade: modificações estéticas e ligadas à saúde, próteses, a recusa em envelhecer e a melhora da performance.

Nesse sentido torna-se evidente a alteração considerável no cenário das grandes empresas de mídia, com a ascensão e valorização de empresas que trabalham a partir desses elementos como a Netflix, Spotify e Facebook, entre outras - cujo poder de engajamento do espectador frente à tela e a desmaterialização do conteúdo, ao lado da onipresença dos softwares e algoritmos, são elementos centrais na sua constituição

Assim, a perspectiva de observação dos fluxos da audiência e da plataforma (JENSEN, 1995; SILVA, MORIGI, 2015; VALIATI, 2018) faz sentido ao considerarmos o ambiente de consumo via streaming: há o fluxo constante de conteúdo nas plataformas streaming (abastecido pela indústria, por meio de aquisições e licenciamentos), a disponibilização em uma sequência planejada (que depende das affordances de cada plataforma); e, além disso, as múltiplas possibilidades de streaming e conteúdo disponivel estimulam o trânsito dos usuários pelos catálogos e por outros serviços de streaming, nos mais variados modelos e variedade de suportes disponiveis (fluxo da audiência).

A compreensão desses aspectos também está ligada ao entendimento dos ritmos espaço-temporais: como as algumas práticas surgem e outras desaparecem, a frequência, duração e sequência; como as práticas são integradas e como as respectivas configurações as amplificam ou destroem (SHOVE; PANTZAR; WATSON, 2012). É importante ressaltar que, nos estudos midiáticos, as coordenadas espaço-temporais são capazes de afetar a nossa experiência com a mídia (SILVERSTONE, 2002). De acordo com Silverstone (2002, p. 161)

$$
\begin{aligned}
& \text { os espaços de engajamento e da experiência } \\
& \text { com a midia são tanto reais como simbólicos } \\
& \text { [.... eles dependem da locação e das rotinas que } \\
& \text { definem as nossas posições no espaço-tempo } \\
& \text { e estas rotinas definem os lugares do, e para } \\
& \text { o, consumo da mídia. }
\end{aligned}
$$

Dessa forma, o consumo em ambiente digital fornece novos contornos a uma multiplicidade de práticas cotidianas das audiências, ou cria novos hábitos e rituais, como clusters de práticas relacionadas (PETERSON, 2010). As plataformas de streaming hoje prezam pela usabilidade e pelo catálogo de conteúdo variado, que não chega a ser ilimitado, mas oferece uma gama ampla de opções. Esse processo, portanto, estimula o aspecto do uso em detrimento da posse, carac- 
terísticos da digitalização - o costume de possuir o material é alterado pelo acesso (PASE, 2010). Deve-se levar em conta que, embora se tenha a ilusão de que o conteúdo estará sempre disponivel, nem sempre se pode contar com isso, em função dos prazos contratuais do licenciamento das obras e do próprio interesse da empresa de manter certos acordos e produções. Além disso, o acesso depende de condições tecnológicas (conexão banda larga) e recursos financeiros (pagamento da mensalidade) e da atividade de cada um nas plataformas de streaming, o que compromete a ideia de acesso ilimitado divulgada em seus discursos promocionais.

A aplicação dessa abordagem em estudos de consumo de mídia no ambiente digital pode ser observada em trabalhos recentes. Montardo (2016), a partir da teoria da prática, propõe uma aproximação do conceito de Warde (2005) ao consumo digital relacionado à performance em sites de redes sociais. Sob essa perspectiva, a autora propõe a conceituação de consumo digital: "o consumo digital consiste no acesso, na produção, na disponibilização e no compartilhamento de conteúdo digital, envolvendo ou não a alocação de recursos financeiros para tanto" (MONTARDO, 2016, p. 10). Ao mesmo tempo, o estudo de Valiati (2018) propõe a utilização da TP para a compreensão do consumo de produtos audiovisuais sob demanda em plataformas digitais, por meio de entrevistas e observação, evidenciando o caráter social e recursivo das ações e a dimensão afetiva que o conteúdo e plataforma assumem na rotina dos usuários.

Warde (2014) aponta que o uso das teorias de prática, são, em parte, uma tentativa de corrigir algumas questões da análise cultural que foi hegemônica durante o segundo período de estudos sobre o consumo. Essa abordagem fornece, portanto, um enquadramento alternativo aos modelos de escolha individual e de um "consumidor soberano" e explora os fenômenos normalmente ocultos na análise cultural do consumo. Um dos aspectos ressaltados pelo autor é a ênfase no papel da rotina, fluxo e sequência e consciência prática. No que diz respeito às críticas, à teoria, elas incluem desde a imprecisão teórica, passando pelo ecletismo metodológico até as dificuldades de sua aplicação ao cenário político e econômico.

Uma das questões enfatizadas por Warde (2014) que podem limitar o uso da TP guarda relação com a identificação das práticas. As formas de desempenho performance são bastante variadas e, com isso, torna-se complicado estabelecer o limite de onde começa e termina a prática. Além disso, como diferenciar atividades pessoais e prática coletivas? Warde (2014) sugere que há pelo menos quatro maneiras de identificar práticas integrativas: a) através da existência de um manual de instruções, delineando como desempenhar uma atividade; b) um número suficiente de pessoas alocando uma quantidade significativa de tempo para uma atividade, e sabendo o que estão realizando; c) a possibilidade de disputas com outros participantes sobre os padrões de performance à luz dos padrões de excelência associados à prática; e d) a possibilidade de identificar conjuntos de equipamentos especializados dedicados a uma atividade. Essas pistas, segundo o autor, constituem um conjunto de indicadores da existência de uma prática que é coletiva em sua prescrição e avaliação. No caso do consumo em ambiente digital via streaming essa tipificação pode ser verificada, mas evidentemente, conta com suas especificidades. O "manual de instruções" na maior parte das plataformas é o conjunto de regras e o passo a passo para a utilização; há uma alocação de tempo mensurável na utilização; em grande parte dos casos, performance é exibida e compartilhada em sites de redes sociais (como por exemplo, quem maratona e comenta a série primeiro; ou a corrida para ouvir uma nova música ou álbum disponivel) e, claramente uma série de equipamentos são comercializados com esta finalidade (os dispositivos de streaming de mídia ou planos de empresas de telefonia que oferecem pacotes que incluem o uso de determinadas plataformas).

Outra questão enfatizada pelo autor diz respeito à regularidade de desempenho das práticas. Sob esse viés, o autor afirma que é possivel considerar que "[...] as performances de um único indivíduo em muitas ocasiões e muitos individuos em 
circunstâncias semelhantes são representações dos procedimentos de uma prática realizada em comum" (WARDE, 2014, p. 292, tradução nossa). ${ }^{6}$ As teorias de prática, de maneira geral têm enfatizado, de maneira útil, o papel do hábito e da rotina em relação ao consumo, mas, no entanto, para o autor, o aparato conceitual alternativo para lidar com performances repetitivas é pouco desenvolvido. Nesse caso, a questão primordial é: como as pessoas passam a ter rotinas ou procedimentos práticos (e temporais) que os levam a repetir atividades mais ou menos similares a outras pessoas em situações semelhantes? As respostas potenciais podem ser variadas, mas, para o autor quaisquer que sejam os mecanismos enfatizados, a regularidade e a ordem identificada pelas teorias da prática surgem de processos baseados em repetição, hábito, rotina e convenção.

Com isso pode-se visualizar uma aproximação entre a TP e as possibilidades da sua utilização para a compreensão do consumo midiático no contexto digital, mais especificamente, do conteúdo via streaming. Ao utilizar a abordagem da prática, busca-se contemplar a compreensão das múltiplas articulações da mídia na vida cotidiana, partindo da ideia de que os atores controlam o fluxo de suas atividades (GIDDENS, 2009), o que caracterizaria um consumidor ativo, com a ampliação do leque de escolhas e controle do tempo possibilitado pelo avanço dos aparatos tecnológicos e, ainda, de uma mudança comportamental em curso. Com esse viés busca-se, portanto, entender o consumo em plataformas de streaming como um fluxo contínuo com diversas intersecções dinâmicas e relacionais, configurando-se como uma dinâmica sociotécnica. 0 modelo a seguir visa identificar os elementos necessários para a análise desse tipo de plataforma a partir desse enquadramento teórico.

\section{Elementos para a análise de consumo em plataformas de streaming}

Como mencionado anteriormente, a utilização da teoria da prática enquanto perspectiva teórica para o estudo do consumo digital, e, neste caso, via streaming pode ser uma lente para ampliar aspectos de processos sociais comuns que geram padrões observáveis de consumo (WARDE, 2014). A esse respeito, ao abordar os desafios metodológicos da utilização da teoria de práticas em pesquisas que abordam o consumo, Halkier e Jensen (2011) sugerem focar o caráter cotidiano, performativo e relacional das práticas e atividades de consumo, de forma a analisar: a) formas de consumir e como elas estão emaranhadas em redes de reprodução e mudança social, em vez de se basearem na análise das escolhas individuais dos consumidores; e b) formas de consumir como realizações dinâmicas e relacionais continuas nas intersecções de múltiplas práticas na vida cotidiana, em vez de analisar o consumo por meio de tipos fixos e superestimar a estabilidade de tais categorias.

Como afirmado anteriormente, a estabilidade em plataformas de streaming é sempre temporária, e refere-se tanto ao aspecto material do serviço quanto às afetações que seus usos têm sobre quem as utiliza e, ainda, às conseqüências desses usos sobre a própria plataforma. Plataformas são "sistemas automatizados que projetam e manipulam conexões" (VAN DIJCK, 2013, p. 12), contemplando a conexão humana e a conectividade automatizada. Isso porque transformam e influenciam o curso da ação, por meio da codificação de atividades sociais em meta(dados) processados por algoritmos e protocolos via interfaces amigáveis baseadas em configurações-padrão de acordo com os interesses estratégicos de seus proprietários (GILLESPIE, 2010).

Couldry (2012), citado por Gillespie (2014), enfatiza que algoritmos são projetados para integrar as práticas, que produzem as informações que eles processam, e, assim, para serem incorporados às rotinas de informação dos usuários. No caso de os usuários não os utilizarem, irão falhar. Por isso, devem ser considerados nos termos de um entrelaçamento multidimensional com as práticas das pessoas. Segundo Gillespie (2014),

\footnotetext{
6 Do original: [...] the performances of both a single individual on many occasions and very many individuals in similar circumstances are enactments of the procedures of a practice held in common.
} 
algoritmos mudam, pessoas moldam o algoritmo com seus usos, e alteram a percepção de si mesmas também devido a isso, de modo que esse ciclo recursivo deve ser apreendido como um todo, levando em conta os cálculos dos algoritmos e os cálculos das pessoas.

Compreender esse ciclo recursivo no consumo de conteúdo em plataformas de streaming pressupõe identificar protocolos técnicos de procedimentos automatizados de personalização previstos nos sistemas de recomendação dessas plataformas e o entrelaçamento disso com o consumo de conteúdos aí disponibilizados. Dessa forma, o modelo de análise de Shove, Pantzar e Watson (2012) a partir dos elementos que compõem a prática: materiais (objetos, tecnologias, entidades fisicamente tangiveis, e coisas das quais os objetos são feitos), competências (habilidades, know-how e técnicas) e significados (que inclui significados simbólicos, ideias e aspirações) pode ser adaptado aos estudos de consumo midiático. É importante ressaltar que estes elementos estão em constante interconexão e são dinâmicos, em constante reinvenção - a prática não existiria com esses elementos isolados.

A observação dos aspectos materiais explora os objetos, a multiplicidade de telas, os algoritmos e sistema de recomendação, as restrições e possibilidades, os recursos financeiros para a manutenção da prática, o próprio corpo e performance (posições, privação de sono, estratégias). Nesse sentido, importa explorar a plataforma de streaming selecionada para caracterizar suas affordances e, com isso, identificar as performances algoritmicas por elas previstas. Essa etapa pressupõe, também, a investigação acerca do conteúdo disponivel na plataforma (música, séries/videos, jogos digitais etc.).

Para fins de descrição de competências, sugere-se apurar os usos da plataforma pelos usuários, visando identificar os processos de individuação aí implicados. Estes aspectos estão ligados às habilidades necessárias para o acesso e navegação, a busca de conteúdo ao desenvolvimento e reconfiguração de capacidades cognitivas e de sensorialidades, como a atenção e a capacidade de consumo do conteúdo em múltiplos suportes, por exemplo, ou, a modificação de dinâmicas da audiência. Outra possibilidade é a análise a partir de perspectiva diacrônica (o consumo antes da utilização da plataforma e o que mudou). É fato que muitas dessas questões relacionam-se diretamente aos aspectos materiais, significados, tempo e interação, haja vista que a prática é formada por um entrelaçamento desses elementos.

Finalmente, importa apreender os significados atribuidos à prática analisada, de forma a localizar impressões sobre si e sobre os outros em função do consumo de determinado conteúdo. A análise da interação social e as dinâmicas relacionais motivadas pelo consumo é pertinente a isso (CHRISTENSEN; ROPKE, 2010; HALKIER; JENSEN, 2011; VALIATI, 2018) - o engajamento com as plataformas (de que forma ela está presente no cotidiano), as implicações no processo de socialização (face a face ou on-line) viabilizados a partir da prática, a produção e utilização de paratextos midiáticos, as indicações e o compartilhamento de conteúdo, a modificação de dinâmicas familiares.

Além disso, destaca-se que as práticas são ordenadas no tempo e espaço (GIDDENS, 2009), e, assim, perceber o espaço no qual ocorrem as atividades e o tempo destinado à prática é também notar a construção simbólica realizada a partir da interação entre os agentes, o tempo e as relações sociais que se transformam com as práticas do cotidiano (CERTEAU, 2014; JACKS; CAPPARELLI, 2006). Ao investigar estes padrões do uso e consumo midiático como parte integrada às práticas cotidianas, pode-se perceber a regularidade com que acontecem e como adquirem um caráter ritualizado.

Com relação às possibilidades e procedimentos para a coleta de dados, a entrevista é a técnica mais comumente utilizada no rol de estudos empíricos que fazem uso da abordagem prática. Em parte, isso pode ser explicado pela ideia de que os atores são dotados da capacidade reflexiva (cognoscitividade), da qual trata Giddens (2009), de entender "o que fazem enquanto fazem". Essa seria uma maneira de ativar a consciência prática de cada indivíduo na descrição dos usos das plataformas no cotidiano. Alinhado a essa ideia 
sugere-se, portanto, buscar procedimentos metodológicos que considerem essa capacidade de descrição e reflexão dos usuários. É importante ressaltar também que a composição da teoria da prática junto a outros métodos e técnicas diversificadas, adequadas ao problema que se quer resolver, pode abarcar o objeto em questão de maneira mais ampla.

\section{Considerações finais}

O consumo de conteúdo em plataformas de streaming é parte da rotina de grande parte dos consumidores e vem alterando as práticas e formas de relacionamento com a midia. A personalização do consumo pretendida pelos seus sistemas de recomendação delineia a natureza modulatória dessa prática tal como apresentada, e é, por conta disso, o eixo a partir do qual podem ser examinadas questões relativas à identidade (individual ou coletiva) num cenário orientado por dados.

A teoria da prática, justamente pela flexibilidade de leituras teóricas apresentadas, torna-se uma opção de enquadramento para auxiliar na apreensão do fenômeno. Ao considerar a articulação de elementos como aspectos materiais, as competências e os significados, aliados às dinâmicas relacionais é possivel ter a dimensão do cenário que envolve plataformas e usuários.

A partir do marco conceitual deste estudo, pode-se depreender os algoritmos e sistemas de recomendações são aspectos já inseridos no campo de práticas relacionadas a plataformas de streaming. A materialidade desses processos, por mais invisiveis que possam parecer ao usuário, acabam por interferir no campo da produção do conteúdo e escolhas dos consumidores. Há que se levar em consideração, sob esse aspecto, as possiveis alterações no campo cultural promovidas pelo processamento de informações algorítmicas, tanto no eixo da produção (o algoritmo não produz o conteúdo, mas indica preferências), quanto do consumo (o reforço aos conteúdos com os quais o usuário está familiarizado). Essas intersecções são capazes de alterar práticas há muito estabelecidas e inserir novos elementos no campo do consumo digital.
É importante ressaltar que a proposição de elementos-chave para a análise não tem a intenção de generalizar padrões a partir de comportamentos e rotinas individuais, mas, a partir disso, compreender como se constroem os fluxos de consumo, marcados pela autonomia do usuário e também pela agência das plataformas. Nesse sentido, apesar de que os elementos destacados no modelo se refiram a plataformas de conteúdo de entretenimento (música, filmes, jogos etc.), pode ser estendido a outras plataformas uma vez que todas elas preveem procedimentos automatizados de personalização (sites de redes sociais, por exemplo). O desafio, nesses casos, parece ser o de identificar recorrências quanto ao acesso de diferentes tipos de conteúdo (vídeos, fotos etc.) na constituição de uma prática.

\section{Referências}

ALILOUPOUR, Nicole. The impact on technology on the entertainment distribuition market: the effects de Netflix and Hulu on Cable Revenue. 2016. 746 p. Tese (Doutorado em Economia) - Scripps College, Claremont. 2016. Disponivel em: https://scholarship.claremont.edu/ scripps_theses/746. Acesso em: 12 fev. 2019.

BARBOSA, Livia. O estudo do consumo nas ciências sociais contemporâneas. In: BARBOSA, Livia; CAMPBELL, Colin (org.). Cultura, Consumo e Identidade. Rio de Janeiro: FGV, 2009.

BRUNO, Fernanda. Máquinas de ver, modos de ser. Vigilância, tecnologia e subjetividade. Porto Alegre: Sulina, 2013.

BURROUGHS, Benjamin Edward. Streaming media: audience and industry shifts in a networked society. 2015. 205 f. Tese (Doutorado em Estudos de Comunicação) - Univesity of lowa, lowa, 2015. Disponivel em: https://ir.uiowa.edu/etd/1833. Acesso em:12 fev. 2019.

CASTAÑEDA, Marcelo. Teorias das Práticas na Análise do Consumo. Ciências Sociais Unisinos, São Leopoldo, v. 46, n. 3. p. 248-255, 23 dez. 2010. http://dx.doi. org/10.4013/csu.2010.46.3.04.

CERTEAU, Michel de. A invenção do cotidiano: Artes de fazer. Petrópolis, RJ: Vozes, 1990.

CHENEY-LIPPOLD, John. We are data. Algorithms and the making of our digital selves. New York: New York University Press, 2017.

CHRISTENSEN, Toke; RØPKE, Inge. Can Practice Theory inspire studies of ICTs in everyday life? In: BRÄUCHLER, Birgit; POSTILL, John (org.). Theorising media and practice. Oxford, Nova York: Berghahn, 2010. p. 233-278. 
COFFEY, Aoife. The impact that music streaming services such as Spotify, Tidal and Apple Music have had on consumers, artists and the music industry itself. 2016. 38 f. Dissertação (Mestrado em Midia Digital Interativa) - Universidade de Dublin, Dublin, 2016. Disponivel em: https://scss.tcd.ie/publications/theses/ diss/2016/TCD-SCSS-DISSERTATION-2016-027.pdf. Acesso em: 12 fev. 2019.

COULDRY, Nick. Theorising Media as a Practice. In: BRÄUCHLER, Birgit; POSTILL, John (org.). Theorising media and practice. Oxford: Berghahn, 2010. p. 35-54.

DATTA, Hannes; KNOX, George; BRONNENBERG, Bart. Changing their tune: How consumer's adoption of online streaming affects music consumption and Discovery. Informs, Catonsville, v. 36, n. 2, p. 1-17, 2017. Disponível em: https://pubsonline.informs.org/doi/10.1287/ mksc.2017.1051. Acesso em: 11 fev. 2019.

GIDDENS, Anthony. A constituição da sociedade. São Paulo: WMF Martins Fontes, 2009.

HAGGEN, Anja; LÜDERS, Marika. Social streaming? Navigating musica as personal and social. Convergence, Catonsville, v. 23, n. 6, p. 1-17, 2016. Disponivel em: https://journals.sagepub.com/doi/ abs/10.1177/1354856516673298. Acesso em: 11 fev. 2019

GILLESPIE, Tarleton. The relevance of algorithms. In: GILLESPIE, Tarleton; BOCZKOVWSKI, Pablo; FOOT, Kirsten. Media Technologies. Essays on communication, materiality, and society. Cambridge: MIT Press, 2014.

HALKIER, Bente; JENSEN, Iben. Methodological challenges in using practice theory in consumption research. Examples from a study on handling nutritional contestations of food consumption. Journal Of Consumer Culture, [S. I], v. 11, n. 1, p. 101-123, mar. 2011. SAGE Publications. Disponivel em: https://journals.sagepub.com/doi/ abs/10.1177/1469540510391365. Acesso em: 10 jan. 2019.

JACKS, Nilda; CAPPARELLI, Sérgio. Etnografia, mídia e cotidiano: eixo sincrônico. In: JACKS, Nilda, CAPPARELLI, Sérgio (org.). TV, familia e identidade: Porto Alegre "fim de século". Porto Alegre: Edipucrs, 2006. p. 165-195.

JENSEN, Klaus. Reception as Flow: Media Constituents of Social Semiotics. In: JENSEN, Klaus. The Social Semiotics of Mass Comunication. London: SAGE PUblications. 1995.

LEMOS, André. A comunicação das coisas: teoria ator-rede e cibercultura. São Paulo: Annablume, 2013.

MAGAUDDA, Paolo. When materiality 'bites back': digital music consumption practices in the age of dematerialization. Journal of Consumer Culture, [S. I.], v. 11, n. 1, p. 15-36, mar. 2011. Disponivel em: http://dx.doi. org/10.1177/1469540510390499. Acesso em: 1 fev. 2018

MONTARDO, Sandra Portella; PAZ; Samyr. Performance Play: Consumo digital como plataforma no streaming de games da plataforma Twitch. Revista Fronteiras. Estudos Midiáticos, São Leopoldo, v. 20, n. 2, p. 190-203, 2018.

MONTARDO, Sandra Portella. Consumo digital e teoria de prática: uma abordagem possivel. Revista FAMECOS, Porto Alegre, v. 23, n. 2, p. 1-15, 2016.
PASE, André. Do papel ao Ipad: livros e revistas publicadas como aplicativos em bibliotecas 2.0. In: CONGRESSO BRASILEIRO DE CIÊNCIAS DA COMUNICAÇÃO, 33., 2010, Caxias do Sul. Anais [...]. Caxias do Sul: UCS, 2010. p. 1-15. Disponivel em: http://www.intercom.org. br/papers/nacionais/2010/resumos/R5-3122-1.pdf. Acesso em: 10 dez. 2018

PAUL, Christiane. From Immateriality to Neomateriality. In: INTERNATIONAL SYMPOSIUM ON ELETRONIC ART, 21, 2015. Proceedings [...]. Disponivel em: https:// isea2015.org/proceeding/submissions/ISEA2015_submission_154.pdf. Acesso em: 1 maio 2020.

PETERSON, Mark Allen. 'But it is my habit to read the Times': metaculture and practice in the reading of Indian newspapers. In: BRÄUCHLER, Birgit; POSTILL, John (org.). Theorising media and practice. Oxford: Berghahn, 2010. p. 127-145

POSTILL, John. Introduction: theorising media and practice. In: BRÄUCHLER, B.; POSTILL, John (org.). Theorising media and practice. Oxford: Berghahn, 2010.

PREY, Robert. Nothing personal: algorithmic individuation on music streaming platforms. Media, Culture \& Society, Londres, v. 40, n. 7, p. 1086-1100, 2018. Disponivel em: https://journals.sagepub.com/doi/ abs/10.1177/0163443717745147. Acesso em: 11 fev. 2019.

RECKWITZ, Andreas. Toward a theory of social practices: a development in culturalist theorizing. European Journal of Social Theory, [S. I.], n. 5, p. 243-63, 2002.

SCHATZKI, Theodore. Introduction: practice theory. In: SCHATZCHI, Theodore; KNORR CETINA, Karin; von SAVIGNY, Eike (org.). The practice turn in contemporary theory. London: Routledge, 2001.

SCHATZKI, Theodore. Social practices: a Wittgensteinian appproach to human activity and the social. Cambridge: Cambridge Univerity Press, 1996.

SHARMA, Romil. The Netflix effect: impacts of streaming model on television storytelling. 2016. 214 p. Tese (Doutorado em Estudos Fílmicos) - Universidade Wesleyan, Middletown, 2016. Disponivel em: https:// wesscholar.wesleyan.edu/etd_hon_theses/1590. Acesso em: 12 fev. 2019

SHOVE, Elizabeth. Everyday practice and the production and consumption of time. In: SHOVE, Elizabeth; TRENTMAN, Frank; WILK, Richard. Time, consuption and everyday life: practice, materiality and culture. Oxford: Berg, 2009.

SHOVE, Elizabeth; PANTZAR, Mika; WATSON, Matt. The dinamics of social practice: everyday life and how it changes. Londres: Sage, 2012.

SILVA, Nathália; MORIGI, Valdir. Fluxo transmidiático: entre as possibilidades de discutir a recepção no ambiente de mídias. Rizoma, Santa Cruz do Sul, v. 3, n. 1, p. 81-93, jul. 2015. Disponivel em: https://online.unisc. $\mathrm{br} / \mathrm{seer} / \mathrm{index} . \mathrm{php} / \mathrm{rizoma/article/view/5330}$. Acesso em: 10 dez. 2017

SILVERSTONE, Roger. Por que estudar a mídia? São Paulo: Loyola, 2002 
SLAGER, Remco. Streaming into the future: the value of music in the digital age. 2016. 46 f. Dissertação (Mestrado em Humanidades) - Universidade de Utrecht, Utrecht, 2016. Disponivel em: https://dspace.library. uu.nl/handle/1874/338317. Acesso em: 12 fev. 2019.

SMITH, Michael; TELANG, Rahul. Streaming, sharing, stealing. Big data and the future of entertainment. Cambridge: The MIT Press, 2016.

THOMES, Tim Paul. An economic analysis of online streaming: how the music industry can generate revenues from cloud computing. Centre for European Economic Research, [S. I.], 2011. Disponivel em: https://papers.ssrn. $\mathrm{com} / \mathrm{sol} 3 /$ papers.cfm?abstract_id=1866228. Acesso em: 1 maio 2020.

THRIFT, Nigel. Beyond Mediation: Three New Material Registers and Their Consequences. In: MILLER, Daniel. Materiality. Durham: Duke University Press, 2005

VALIATI, Vanessa. "Você ainda está assistindo?": O consumo audiovisual sob demanda em plataformas digitais e a articulação das práticas relacionadas à Netflix na rotina dos usuários. 278 f. Tese (Doutorado em Comunicação e Informação) - Universidade Federal do Rio Grande do Sul, Porto Alegre, 2018. Disponivel em: https://lume.ufrgs.br/handle/10183/174831\#. Acesso em: 10 jan. 2019.

VAN DIJCK, José. The culture of connectivity. A critical history of social media. Nova York: Oxford University Press, 2013

VONDERAU, Patrick. The Politics of Content Aggregation. Television \& New Media, [S. I.], v. 16, n. 8, p. 717-733, 2015.

ZUILHOF, Jan. The soundtracked self: algorithm individuation on Spotify. 2014. 58 p. Dissertação (Mestrado em Nova Midia e Culturas Digitais) - Universidade de Amsterdan, Amsterdan, 2014. Disponivel em: https://Www.google.com/url?sa= $t \& r c t=j \& q=\& e s r c=s \& s$ ource $=w e b \& c d=1 \& v e-$ d=2ahUKEwiP47z_ibbgAhUpH7kGHVgWAgoQFjAAe gQICRAC\&url=http \%3A\%2F\%2Fscriptiesonline. uba.uva.nl\%2Fdocument\%2F605922\&usg=AOvVawo gd7ba88zlyHBgt7FToPoD. Acesso em: 12 fev. 2019.

WARDE, Alan. After taste: Culture, consumption and theories of practice. Journal Of Consumer Culture, [S. I.], v. 14, n. 3, p. 279-303, 25 ago. 2014. Disponivel em: https:// journals.sagepub.com/doi/10.1177/1469540514547828. Acesso em: 10 jan. 2019.

WARDE, Alan. Consumption and Theories of Practice. Journal of Consumer Culture, [S. I.], v. 5, n. 2, p. 131153, jul. 2005. Disponivel em: https://journals.sagepub. $\mathrm{com} / \mathrm{doi} / 10.1177 / 1469540505053090$. Acesso em: 10 dez. 2018

\section{Sandra Portella Montardo}

Doutora em Comunicação Social pela Pontificia Universidade Católica do Rio Grande do Sul (PUCRS), em Porto Alegre, RS, Brasil; professora e pesquisadora da Universidade Feevale (PPG em Processos e Manifestacões Culturais, PPG em Diversidade Cultural e Inclusão Social e Mestrado Profissional em Indústria), em Novo Hamburgo, RS, Brasil.

\section{Vanessa Amália Dalpizol Valiati}

Doutora em Comunicação e Informação pela Universidade Federal do Rio Grande do Sul (UFRGS), em Porto Alegre, RS, Brasil; professora e pesquisadora da Universidade Feevale (Mestrado Profissional em Indústria Criativa), em Novo Hamburgo, RS, Brasil.

\section{Endereço para correspondência}

Sandra Portella Montardo/ Vanessa Amália Dalpizol Valiati

Universidade Feevale

Campus II, RS-239, 2755

Vila Nova, 9335-2000

Novo Hamburgo, RS, Brasil

Os textos deste artigo foram revisados pela Poá Comunicação e submetidos para validação do(s) autor(es) antes da publicação. 